\title{
INFLUENCIA DEL CUIDADO ESPIRITUAL QUE BRINDA EL PROFESIONAL DE ENFERMERÍA EN EL ESTADO DE ÁNIMO, NIVEL DE COLABORACIÓN Y SIGNOS VITALES DE LOS PACIENTES HOSPITALIZADOS EN LA UNIDAD DE CUIDADOS INTENSIVOS DEL HOSPITAL NACIONAL GUILLERMO ALMENARA IRIGOYEN, MARZO - ABRIL, 2009
}

\author{
Gloria Orietta BULLÓN LÓPEZ1', María luisa GABINO FABIÁN²
}

\begin{abstract}
RESUMEN
Objetivo: Determinar la influencia del cuidado espiritual que brinda el profesional de enfermería en el estado de ánimo, nivel de colaboración y signos vitales de los pacientes hospitalizados en la Unidad de Cuidados Intensivos del Hospital Nacional Guillermo Almenara Irigoyen. Metodología: El diseño del estudio fue cuantitativo, de tipo cuasi experimental, pues se trabajó con dos grupos de pacientes, un grupo experimental al cual se le brindó el cuidado espiritual y un grupo control al que no se le brindó este cuidado. Previamente, se capacitó a 8 enfermeras para que participen en el cuidado espiritual, haciendo uso de un módulo "Cuidado Espiritual al Paciente Crítico" elaborado por las autoras. Como instrumento de recolección de datos, se utilizó una guía de observación cuya validez fue medida a través de juicio de expertos y la confiabilidad mediante la prueba de Cronbach's. Los datos recolectados fueron procesados utilizando el sofware estadístico SPSS 15.0. Resultados: En el grupo experimental, antes de recibir el cuidado espiritual el $60 \%$ de los pacientes presentó un estado de ánimo malo y el $30 \%$ un estado de ánimo bueno. Después de recibir el cuidado espiritual el $100 \%$ presentó un estado de ánimo entre muy bueno y bueno. En el grupo control, en la evaluación inicial el $100 \%$ de los pacientes presentó un estado de ánimo entre malo y muy malo, en la evaluación final el 100\% presentó un estado de ánimo malo. En el grupo experimental antes de recibir el cuidado espiritual, el $60 \%$ de los pacientes presentó un nivel de colaboración malo y el 30\%, un nivel bueno; después de recibir el cuidado espiritual el 100\% presentó un nivel de colaboración entre muy bueno y bueno. En el grupo control, en la evaluación inicial el $80 \%$ de los pacientes presentó un nivel de colaboración malo y en la evaluación final, el 50\% presentó un nivel de colaboración malo y otro porcentaje igual un nivel de colaboración bueno. En el grupo experimental, el 90\% no modifica sus funciones vitales después de recibir el apoyo espiritual y en el grupo control el 70\% tampoco modifica sus funciones vitales en la evaluación final. Sin embargo, se observa que el $30 \%$ del grupo control mejora sus funciones vitales y sólo un 10\% lo hace en el grupo experimental. Conclusiones: El cuidado espiritual influye positivamente en el estado de ánimo y nivel de colaboración de los pacientes de la UCI con un nivel de significancia de 0.000 para el grupo experimental. Sin embargo, no tiene influencia en las funciones vitales de los pacientes de la UCI, alcanzando un nivel de significancia mayor de 0.05 en el grupo experimental.
\end{abstract}

Palabras clave: Influencia, cuidado espiritual, estado de ánimo, nivel de colaboración, signos vitales.

\begin{abstract}
Objective: To determine the influence of spiritual care provided by nursing professionals in the mood, level of cooperation and vital signs of patients hospitalized in the Intensive Care Unit at the Guillermo Almenara Irigoyen General Hospital. Methodology: The study design was quantitative, quasi-experimental, because it worked with two groups of patients, an experimental group which was provided with spiritual care and a control group that was not given this type of care. Previously, 8 nurses were trained to participate in the spiritual care, using a module " Spiritual Care for the Critical Patient " developed by the authors. As a data collection instrument, an observation guide was used which validity was measured through expert opinion and reliability using Cronbach's test. The collected data were processed using the SPSS 15.0 statistical software. Results: In the experimental group, prior to receiving spiritual care, $60 \%$ of patients showed a bad mood and $30 \%$ a good mood. After receiving spiritual care
\end{abstract}

1 Licenciada en Enfermería, Especialista en Cuidados Intensivos, Enfermera del Hospital Guillermo Almenara

2 Licenciada en Enfermería, Especialista en Cuidados Intensivos, Enfermera del Hospital Casimiro Ulloa 
the $100 \%$ of patients in the experimental group had a mood between very good and good. In the control group in an initial assessment $100 \%$ of the patients had a mood between bad and very bad in the final evaluation; $100 \%$ had a poor mood. In the experimental group prior to receiving spiritual care, $60 \%$ of the patients showed a bad level of cooperation and $30 \%$ a good level. After receiving spiritual care the $100 \%$ of patients had a level of cooperation between very good and good. In the control group in the initial evaluation $80 \%$ of patients had a bad cooperation level. In the final evaluation $50 \%$ showed a poor level of cooperation and the same percentage showed a good level of cooperation. In the experimental group, $90 \%$ did not modify their vital functions after receiving spiritual support. In the control group $70 \%$, in the same way, did not modify their vital functions in the final evaluation. However, it is observed that $30 \%$ of the control group improved their vital functions and only $10 \%$ improved in the experimental group. Conclusions: Spiritual care positively affects mood and level of cooperation of patients in the ICU with a significance level of 0.000 for the experimental group. However, it does not have influence on the vital functions of patients in the ICU, reaching a level of significance greater than 0.05 in the experimental group.

Key Words: Influence, spiritual care, mood, level of cooperation, vital signs.

\section{INTRODUCCIÓN}

Lo más relevante de la enfermería como arte y disciplina, según refiere Watson (1985), es la institucionalización del cuidado como piedra angular y objetivo central. Es por esta razón que el cuidado debe estar constituido de acciones que permitan proteger, mejorar y preservar la humanidad ayudando a la persona a encontrar un significado a su enfermedad, sufrimiento, dolor y existencia, para que de esta forma adquiera autocontrol, autoconocimiento y autocuración.

Cuidar en sentido holístico significa considerar a la persona en todas sus dimensiones: física, intelectual, social, emocional, espiritual y religiosa. De esta forma, el cuidado de enfermería pasa de cuidar el cuerpo a cuidar a la persona en la experiencia de la salud - enfermedad. A través de este tipo de cuidado, el profesional de enfermería ayuda al paciente a experimentar bienestar dentro de su enfermedad, a recuperarse o tener una muerte en paz y con dignidad.

El cuidado espiritual, como parte del cuidado holístico ha sido reconocido muy útil en la salud de las personas, pues como menciona Koening (2002), la oración o la meditación son consideradas como medidas terapéuticas que pueden intervenir en la recuperación. Asimismo, Álvarez (2007), afirma que "rezar por el paciente y con el paciente puede ser un alivio para ellos y para nosotros mismos”.

La espiritualidad, en cada persona, es considerada también como fuente de fortaleza para asumir la enfermedad, pues le provee armas para darle sentido al sufrimiento para que así éstas influyan en la modificación de su estado de ánimo, que al mejorar, le permitirá tomar decisiones sobre su participación en la recuperación de su salud y en su respuesta al tratamiento (Puchalski, 2006).
En general, las prácticas espirituales se asocian con la disminución de la morbi mortalidad, menor depresión, mejores hábitos y menor estancia hospitalaria, pues el paciente se empodera de su situación de enfermedad y tiene mejor disposición para enfrentarla (Koening, 2002; Prado, 2007).

En la Unidad de Cuidados Intensivos, debido al estado de complejidad vital en la que llegan los pacientes, muchas veces hace que la atención se centre en la dimensión física, dejando en un plano secundario las dimensiones psicológica, social y espiritual, olvidando que la espiritualidad ofrece al paciente crítico un marco de devoción estructural que proporciona alivio y consuelo en los momentos de dolor, soledad, desesperanza e incertidumbre que se generan al estar en estado crítico o cerca a la muerte (Fernández, 1998). Para que los profesionales de enfermería de la UCI puedan brindar el cuidado espiritual, deben explorar e identificar lo que es significativo en la vida de cada paciente y las formas individuales que tienen de ajustarse al dolor y al sufrimiento. Además, deben conocer cómo las creencias religiosas influyen en la emocionalidad de las personas (Mc Sheerry, 1998).

En la práctica profesional, se ve con preocupación que no siempre los principios del cuidado espiritual son aplicados, ya que las instituciones hospitalarias muchas veces están impregnadas del modelo biomédico y curativo que reduce lo humano a lo biológico alejando el trabajo de la enfermera de su visión humanista y holística del cuidado (Poblete, 2006).

La motivación para realizar este estudio fue el apreciar que en el diario quehacer dentro de la UCI del HNGAI se despliega un gran trabajo tecnológico y biomédico con cada paciente allí internado, los profesionales de enfermería debidamente capacitados brindan el cuidado 
diario de acuerdo a la priorización de necesidades utilizando formatos de valoración y planificación de actividades en las que no se incluyen aspectos de la esfera espiritual del paciente, a pesar de que en forma individual muchos de estos profesionales realizan este cuidado que da el sentido humanístico a la profesión pues la espiritualidad de la persona es muchas veces la motivación más fuerte para luchar por la vida, sobre todo en un paciente críticamente enfermo. Es por eso que con el deseo de revalorar la profesión de enfermería al realizar el cuidado al paciente en ésta área que a veces postergamos a pesar de tener gran importancia, es por este motivo que se realizó el presente estudio, en base a lo observado y aportando un instrumento para que el profesional de enfermería pueda realizar la valoración de las necesidades espirituales y planificar su intervención.

El objetivo del presente estudio de investigación es determinar cómo influye el cuidado espiritual que brinda el profesional de enfermería en el estado de ánimo, nivel de colaboración y signos vitales de los pacientes de la Unidad de Cuidados Intensivos del Hospital Nacional Guillermo Almenara Irigoyen.

\section{MATERIAL Y MÉTODOS}

El presente estudio se realizó en la Unidad de Cuidados Intensivos del Hospital Nacional Guillermo Almenara Irigoyen, durante el mes de abril del 2009. La muestra estuvo conformada por 20 pacientes, seleccionada a través del muestreo no probabilístico, por conveniencia, teniendo como criterios de inclusión: pacientes entre 25 y 75 años, con Glasgow de 15 puntos o Glasgow de 11+TET o TQT, sin problemas mentales y con permanencia mínima de 1 semana.

El diseño del estudio fue cuantitativo, de tipo cuasi experimental, pues se trabajó con dos grupos de pacientes, un grupo experimental al cual se le brindó el cuidado espiritual y un grupo control al que no se le brindó este cuidado.

Previamente se capacitó a 8 enfermeras, haciendo uso de un módulo "Cuidado Espiritual al Paciente Crítico" elaborado por las autoras.
Como instrumento de recolección de datos, se empleó una guía de observación validada por el juicio de expertos y sometida a la prueba de confiabilidad de Cronbach’s, con una puntuación de 0.885.

La guía incluye datos generales y datos específicos donde se considera 10 ítemes correspondientes al estado de ánimo, 3 ítemes correspondientes al nivel de colaboración y 3 ítemes correspondientes a las funciones vitales. Cada respuesta Sí equivalía a 2 puntos y cada respuesta NO equivalía a 0 puntos.

El estado de ánimo y nivel de colaboración se midieron utilizando la escala: muy malo, malo, bueno y muy bueno Donde:

- Respuesta SI en 0 días de los 3 días de observación = muy malo (0 puntos).

- Respuesta SI en 1 días de los 3 días de observación $=$ malo (2 puntos).

- Respuesta SI en 2 días de los 3 días de observación = Bueno (4 puntos).

- Respuesta SI en 3 días de los 3 días de observación $=$ Muy bueno (6n puntos).

Para funciones vitales:

- En los 3 días de observación no hay cambio favorable o sólo en 1 día de observación hay cambio favorable $=$ No modifica ( 2 puntos)

- En 2 ó 3 días de observación hay cambio favorable = Mejora (4 puntos).

A los pacientes que conformaron el grupo control, se les aplicó la misma guía al iniciar y finalizar cada turno, durante tres turnos. Las pruebas estadísticas utilizadas fueron: $\mathrm{T}$ de student de comparación de medias para muestra apareadas y T de student de comparación de medias para muestras independientes.

En cuanto a las consideraciones éticas, se respetó en todo momento la confidencialidad de los datos recolectados. 


\section{RESULTADOS}

Tabla 1. Estado de ánimo de los pacientes que conforman el grupo experimental antes y después de recibir el cuidado espiritual por las enfermeras y de los pacientes del grupo control de la UCI del Hospital Nacional Guillermo Almenara Irigoyen, abril, 2009

\begin{tabular}{lcccccccc}
\multicolumn{3}{c}{} & \multicolumn{3}{c}{ Grupo experimental } & \multicolumn{3}{c}{ Grupo control } \\
$\begin{array}{l}\text { ESTADO } \\
\text { DE ÁNIMO }\end{array}$ & \multicolumn{2}{c}{ ANTES } & DESPUÉS & $\begin{array}{c}\text { Evaluación } \\
\text { inicial }\end{array}$ & $\begin{array}{c}\text { Evaluación } \\
\text { final }\end{array}$ \\
& $\mathrm{n}$ & $\%$ & $\mathrm{n}$ & $\%$ & $\mathrm{n}$ & $\%$ & $\mathrm{n}$ & $\%$ \\
\hline Muy malo & 0 & 0 & 0 & 0 & 3 & 15.0 & 0 & 0 \\
Malo & 12 & 60.0 & 0 & 0 & 17 & 85.0 & 20 & 100.0 \\
Bueno & 6 & 30.0 & 3 & 15.0 & 0 & 0 & 0 & 0 \\
Muy bueno & 2 & 10.0 & 17 & 85.0 & 0 & 0 & 0 & 0 \\
\hline \multicolumn{1}{c}{ TOTAL } & 20 & 100.0 & 20 & 100.0 & 20 & 100.0 & 20 & 100.0 \\
\hline
\end{tabular}

La tabla 1 muestra que en el grupo experimental antes de recibir el cuidado espiritual, el $60 \%$ de los pacientes presentó un estado de ánimo malo, el $30 \%$ un estado de ánimo bueno y sólo el 10\% un estado de ánimo muy bueno. Después de recibir el cuidado espiritual, el 100\% de los pacientes presentó un estado de ánimo entre muy bueno y bueno.

En el grupo control, se observa que en la evaluación inicial, el 100\% de los pacientes presentó un estado de ánimo entre malo y muy malo y en la evaluación final, el $100 \%$ presentó un estado de ánimo malo.

Tabla 2. Prueba T de Student de comparación de medias para muestras apareadas en el estado de ánimo de los pacientes que conforman el grupo experimental antes y después de recibir el cuidado espiritual por las enfermeras y de los pacientes del grupo control de la UCI del Hospital Nacional Guillermo Almenara Irigoyen, abril, 2009.

\begin{tabular}{|c|c|c|c|c|c|}
\hline $\begin{array}{c}\text { Variable: } \\
\text { Estado de ánimo }\end{array}$ & Momento & Promedio & $\mathrm{T}$ & g.l. & Sig \\
\hline \multirow{2}{*}{$\begin{array}{c}\text { Grupo } \\
\text { experimental }\end{array}$} & Antes & 20.60 & \multirow{2}{*}{-7.115} & \multirow{2}{*}{19} & \multirow{2}{*}{0.000} \\
\hline & Después & 49.70 & & & \\
\hline \multirow{2}{*}{ Grupo control } & $\begin{array}{l}\text { Evaluación } \\
\text { inicial }\end{array}$ & 4.80 & \multirow{2}{*}{-1.707} & \multirow{2}{*}{19} & \multirow{2}{*}{0.104} \\
\hline & $\begin{array}{l}\text { Evaluación } \\
\text { final }\end{array}$ & 7.00 & & & \\
\hline
\end{tabular}

En la tabla 2 se observa que el promedio en el estado de ánimo del grupo experimental antes de recibir el cuidado espiritual es de 20.60 y después de recibir el cuidado espiritual es de 49.70. Al aplicar la prueba de comparación de medias para muestras apareadas, se confirma que existe diferencia significativa en el estado de ánimo de los pacientes antes y después de recibir el cuidado espiritual, con un nivel de significancia de 0.000. En cambio en el grupo control, se demuestra que no existe diferencia significativa, mediante un nivel de significancia obtenido de 0.104. 
Tabla 3. Nivel de colaboración de los pacientes que conforman el grupo experimental antes y después de recibir el cuidado espiritual por las enfermeras y de los pacientes del grupo control de la UCI del Hospital Nacional Guillermo Almenara Irigoyen, abril, 2009

\begin{tabular}{lcccccccc}
\multicolumn{1}{c}{$\begin{array}{c}\text { NIVEL DE } \\
\text { COLABORACIÓN }\end{array}$} & \multicolumn{2}{c}{ ANTES } & DESPUÉS & $\begin{array}{c}\text { Gvaluación } \\
\text { inicial }\end{array}$ & $\begin{array}{c}\text { Evaluación } \\
\text { final }\end{array}$ \\
& $\mathrm{n}$ & $\%$ & $\mathrm{n}$ & $\%$ & $\mathrm{n}$ & $\%$ & $\mathrm{n}$ & $\%$ \\
Muy malo & 0 & 0 & 0 & 0 & 0 & 0 & 0 & 0 \\
Malo & 12 & 60.0 & 0 & 0 & 16 & 80.0 .0 & 10 & 50.0 \\
Bueno & 6 & 30.0 & 1 & 5.0 & 4 & 20 & 10 & 50.0 \\
Muy bueno & 2 & 10.0 & 19 & 95.0 & 0 & 0 & 0 & 0 \\
\multicolumn{1}{c}{ TOTAL } & 20 & 100.0 & 20 & 100.0 & 20 & 100.0 & 20 & 100.0 \\
\hline
\end{tabular}

En la tabla 3 se observa que en el grupo experimental antes de recibir el cuidado espiritual, el $60 \%$ de los pacientes presentó un nivel de colaboración malo, el 30\% un nivel de colaboración bueno y sólo el 10\% un nivel de colaboración muy bueno. Después de recibir el cuidado espiritual, el 100\% de los pacientes presentó un nivel de colaboración entre muy bueno y bueno.

En el grupo control se evidencia que en la evaluación inicial el 80\% de los pacientes presentó un nivel de colaboración malo y el 20\% un nivel de colaboración bueno. En la evaluación final el 50\% presentó un nivel de colaboración malo y otro porcentaje igual un nivel de colaboración bueno.

Tabla 4. Prueba $\mathbf{T}$ de Student de comparación de medias para muestras apareadas en el nivel de colaboración de los pacientes que conforman el grupo experimental antes y después de recibir el cuidado espiritual por las enfermeras y de los pacientes del grupo control de la UCI del Hospital Nacional Guillermo Almenara Irigoyen, abril, 2009

\begin{tabular}{|c|c|c|c|c|c|}
\hline $\begin{array}{c}\text { Variable: } \\
\text { Nivel de } \\
\text { colaboración }\end{array}$ & Momento & Promedio & $\mathrm{T}$ & g.l. & Sig \\
\hline $\begin{array}{c}\text { Grupo } \\
\text { experimental }\end{array}$ & $\begin{array}{c}\text { Antes } \\
\text { Después }\end{array}$ & $\begin{array}{c}9.70 \\
16.70\end{array}$ & -6.308 & 19 & 0.000 \\
\hline Grupo control & $\begin{array}{l}\text { Evaluación } \\
\text { inicial } \\
\text { Evaluación } \\
\text { final }\end{array}$ & 4.70 & -3.047 & 19 & 0.070 \\
\hline
\end{tabular}

Fuente: instrumento aplicado a los enfermeros de la UCI del HNGAI, 2009

La tabla 4 muestra que el promedio obtenido por el grupo experimental en el nivel de colaboración antes y después de recibir el cuidado espiritual fue de 9.70 y 16.70 respectivamente. Al aplicar la prueba de comparación de medias para muestras apareadas, se confirma que existe diferencia significativa en el nivel de colaboración de los pacientes antes y después de recibir el cuidado espiritual, con un nivel de significancia obtenido de 0.000 . Por el contrario, se demuestra que en el grupo control no existe diferencia significativa, con un nivel de significancia de 0.070.

Tabla $N^{\circ}$ 5. Funciones vitales de los pacientes que conforman el grupo experimental después de recibir el cuidado espiritual y del grupo control en la evaluación final realizada por las enfermeras de la UCI del Hospital Nacional Guillermo Almenara Irigoyen, abril 2009.

\begin{tabular}{|ccccc|}
\hline NIVEL DE & \multicolumn{2}{c}{ Grupo experimental } & \multicolumn{2}{c|}{ Grupo control } \\
COLABORACIÓN & $\mathbf{n}$ & $\%$ & $\mathbf{n}$ & $\%$ \\
\hline No modifica & 18 & 90.0 & 14 & 70,0 \\
Mejora & 2 & 10.0 & 6 & 30,0 \\
\hline \multicolumn{1}{c}{ TOTAL } & 20 & 100.0 & 20 & 100.0 \\
\hline
\end{tabular}

Fuente: instrumento aplicado a los enfermeros de la UCI del HNGAI, 2009 
En la tabla 5 se observa que en el $90 \%$ de pacientes que conforman el grupo experimental no modifican sus funciones vitales después de recibir el apoyo espiritual y sólo un 10\% mejoran sus funciones vitales; en el $70 \%$ de pacientes que conforman el grupo control tampoco modifican sus funciones vitales en la evaluación final y el 30\% mejora sus funciones vitales.

Tabla 6. Prueba de Comparación de medias para muestras independientes de las funciones vitales entre grupo experimental y el grupo control de pacientes de la UCI del Hospital Nacional Guillermo Almenara Irigoyen, Abril, 2009

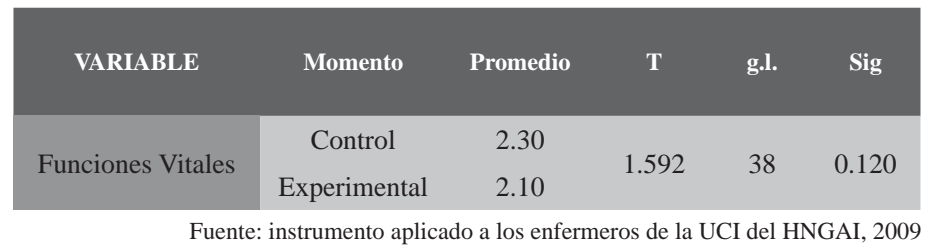

La tabla 6 muestra que el promedio de las funciones vitales del grupo control y el grupo experimental es de 2.30 y 2.10 respectivamente, $\mathrm{y}$ al aplicar la prueba $\mathrm{T}$ comparación de medias para muestras independientes, se confirma que no existe diferencia significativa entre el grupo experimental y control respecto al promedio de las funciones vitales, con un nivel de significancia de 0.12.

\section{DISCUSIÓN Y CONCLUSIONES}

Al concluir la investigación se encontró que la mayoría de los pacientes del grupo experimental antes de recibir el cuidado espiritual presentó un estado de ánimo malo, y después de recibirlo, todos presentaron un estado de ánimo entre muy bueno y bueno, lo que no ocurrió con el grupo control al cual no se le brindó dicho cuidado. El estado de ánimo inicial que mostraron los pacientes del estudio, puede explicarse porque según Perdomo (1998), al ingresar ellos a la UCI, atraviesan una crisis existencial, que los aparta de su mundo exterior e interior y altera cada aspecto de sus vidas incluyendo el aspecto espiritual, imprescindible para la paz interior. Toda esta vivencia, se ve reflejada en el estado de ánimo que es definido por Barlow (2003), como una emoción generalizada y persistente que influye en la percepción del mundo y Strongone (2005), agrega que cuando el estado de ánimo cambia, la percepción del mundo también cambia con él, de tal manera que si el estado de ánimo es negativo, la percepción del entorno también será negativa e influirá en la conducta, colaboración y participación de la persona, quien no tendrá disposición para enfrentar la enfermedad de manera positiva. Esto concuerda con los resultados obtenidos en cuanto al nivel de colaboración, pues la mayoría de los pacientes que conformaron el grupo experimental antes de recibir el cuidado espiritual, presentó un nivel de colaboración malo, y lo mismo ocurrió en el grupo control; sin embargo, después de recibir el cuidado espiritual, el grupo experimental en su totalidad cambió a un nivel de colaboración bueno y muy bueno, lo que no ocurrió en el grupo control pues, sólo la mitad presentó un nivel de colaboración bueno y la otra mitad continuó con un nivel de colaboración malo.
Esto quiere decir que el estado de ánimo malo que presentaron ambos grupos de pacientes, probablemente haya influido en su nivel de colaboración, lo que se constituye en un factor negativo para su recuperación, pues puede disminuir su motivación para salir de su situación crítica, aumentando el tiempo de estancia en la UCI, el riesgo de complicaciones y el gasto familiar.

En cuanto a los resultados positivos obtenidos en el estado de ánimo y nivel de colaboración de los pacientes que conformaron el grupo experimental después de recibir el cuidado espiritual, es explicado por Chochinov (2005), quien refiere que la espiritualidad tiene influencia en el paciente para tener pensamientos positivos, mejorar su disposición y colaboración, haciéndolo partícipe activo de su cuidado.

Esta colaboración del paciente hace mucho más humana la práctica de enfermería, pues permite reconocer al otro como persona, con el derecho y la obligación de ser actor de su salud y no simplemente el receptor de actividades planeadas por el profesional que lo cuida. Además, Barrera y Miranda (2000), mencionan que la participación del paciente le permite tomar parte en su cuidado apropiándose del mismo, con la orientación del profesional de enfermería. Dicha participación puede hacerse evidente a diferentes niveles que van desde la información y realización de actividades básicas hasta la toma de decisiones, permitiendo el derecho a estar informado, opinar, proponer y rehusar determinadas actividades, procedimientos y tratamientos. Son varios los motivos para que la espiritualidad, vivida de manera religiosa, sea útil en la salud; entre ellos, se ha 
reconocido que la oración y la meditación generan un estado de paz interna que ayuda a la recuperación, y que el tener pensamiento positivo es terapéutico (Chochinov, 2005).

En cuanto a la variable de las funciones vitales, se obtuvo que la mayoría de pacientes del grupo experimental después de recibir el cuidado espiritual no modificarán sus funciones vitales, en cambio en el grupo control casi las tres cuartas partes de los pacientes mejoraron sus funciones vitales. Estos resultados difieren a la experiencia narrada por la Dra. Dopico da Silva (2002), pues ella refiere que vivió la experiencia dolorosa de tener a su hermano gravemente enfermo, que a pesar del tratamiento médico no evidenciaba mejoría alguna, ella diariamente lo acompañaba, oraba por el y le hablaba de Dios, le pedía que tuviera fortaleza y que se confiara a su Salvador, con los días empezaron a haber cambios en su frecuencia cardiaca que fue mejorando, de igual manera su presión arterial; ella tuvo la convicción de que el sufrimiento había pasado para llevar la enfermedad con esperanza y en paz interna.

En el presente estudio, probablemente hayan influido ciertas variables intervinientes que no fueron controladas, como las características individuales de los pacientes, el uso de medicamentos que tienen efectos sobre las funciones vitales, el tiempo corto del estudio, etc.

La influencia positiva del cuidado espiritual en el estado de ánimo y nivel de colaboración evidenciado en los pacientes del estudio, refuerzan la importancia del mismo en las unidades críticas como parte del cuidado integral de enfermería, pues como mencionan Ankeny, 2005; Ackley \& Bermejo (2005;2004) no sólo es de competencia del capellán como se consideró en el pasado, sino que ha sido aceptado como un enfoque legítimo de la práctica de enfermería.

Aunque las unidades de cuidado crítico son un lugar desafiante para el cuidado espiritual, tal cuidado puede ser una manera de mejorar el carácter curativo y humanitario del ambiente altamente técnico y orientado a lo fisiológico de las VCIs (Bermejo, 2005).

\section{RECOMENDACIONES}

Se recomienda brindar un cuidado holístico a los pacientes considerando como una fuente de fortaleza y de nexo importante entre ellos y los profesionales de enfermería la espiritualidad del paciente que está presente en todo ser humano independientemente de su credo o religión, haciendo que el trance de la enfermedad sea asumido de una forma diferente y que permita a los pacientes liberar sus temores y buscar formas de afrontar la enfermedad y la muerte.

Se recomienda también realizar la revisión del módulo de Cuidado Espiritual elaborado por las autoras del presente estudio, para enriquecerlo y aplicarlo en las áreas críticas como parte del cuidado integral.

\section{REFERENCIAS BIBLIOGRÁFICAS}

1. Alvarez Erick, Cuidado Espiritual/Compendio de Enfermería; 2007.

2. Ankeny RA, Clifford R, Jordens CF, Kerridge IH, Benson R. Augst. Religious perspectives on withdrawal of treatment from patients with multiple organ failure. Med; 2005.

3. Balboni TA, Vanderwerker LC, Block SD. Religiousness and spiritual support among advanced cancer patients and associations with end-of-life treatment preferences and quality of life. J Clin Oncol; 2007.

4. Barlow D.. Enfoque Integral de Psicología anormal; 2003.

5. Barbero J.. Sufrimiento y responsabilidad moral. En Monografías Humanitas $N^{\circ}$ 2. Dolor y Sufrimiento en la Práctica Clínica. Fundación Medicina y Humanidades Médicas, Barcelona, España; 2004.
6. Barrera, Lucy, y Pinto, Natividad. Comparación entre el bienestar espiritual y la habilidad de cuidado de los cuidadores de niños en situación de enfermedad crónica, Facultad de Enfermería, Universidad Nacional de Colombia; 2002.

7. Barrera O y Miranda N. El concepto de participación en el cuidado de enfermería. Trabajo de grado. Pontificia Universidad Javeriana. Facultad de Enfermería. Bogotá; 2000.

8. Bermejo JC.. Espiritualidad y mayores. Red Latinoamericana de Gerontología; 2008.

9. Burkhardt MA.. Spirituality: An Analysis of the Concept. Holistic Nursing Practitioner; 1999.

10. Coyle, J.. Spirituality and health: towards a framework for exploring the relationship between spirituality and health, Journal of Advanced Nursing; 2002. 
11. Curlin FA, Chin MH, Sellergren SA, Roach CJ, Lantos JD.. The association of physicians' religious characteristics with their attitudes and selfreported behaviors regarding religion and spirituality in the clinical encounter. Med Care l; 2006.

12. Chacón, Consuelo y col.. El estado del arte del cuidado de enfermería. Santafé de Bogotá; 2000.

13. E. Chirinos E; Strongone Restrepo. Emociones y estados de Animo.Coaching Ontológico y Organizacional; 2005.

14. Chochinov HM, Cann BJ.. Interventions to enhance the spiritual aspects of dying. Journal of Palliative Medicine; 2005.

15. Chochinov HM. Dying, dignity, and new horizons in palliative end-of-life care. CA Cancer J Clin.; 2006.

16. Emblen JD.. Religion and spirituality defined according to current use in nursing literature. J Prof Nurs; 1998.

17. Fernández, M., Maldonado, l., Rodríguez, g., y Salinas, F.. Manejo de las necesidades emocionales y espirituales de los pacientes hospitalizados en el servicio de Medicina del Hospital Central de Maracay por parte de la enfermera. Trabajo Especial de Grado para optar al título de Licenciado en Enfermería de la Universidad Nacional; 1997.

18. Goddard NC.. Spirituality as integrative energy: A philosophical analysis as requisite precursor to holistic nursing practive. Journal of Advanced Nursing; 1998.

19. Koenig, Harold. An 83-Year-Old Woman with Chronic Illness and Strong Religious Beliefs, JAMA; jul. 24/31 2002, 288.
20. Koenig, Harold. Spirituality in Patient Care: Why, How, When, and What, London, Templeton Foundation Press; 2002.

21. Penna Guimarães Elio \& Álvaro Avezum. Impacto de La Espiritualidad en La salud física. Brasil; 2008.

22. Pinto, Natividad. Comparación del empleo de dos escalas de bienestar espiritual en cuidadores familiares de niños con enfermedad crónica. Trabajo de promoción a profesora asociada, Facultad de Enfermería, Universidad Nacional de Colombia; 2002.

23. Prado Flores. Agencia española de desarrollo spiritual, artículo publicado en España; 2007.

24. Puchalski CM, Lunsford B, Harris MH, Miller RT. Interdisciplinary spiritual care for seriously ill and dying patients: a collaborative model. Cancer J.; 2006.

25. Reed PG.. Developmental resources and depression in the elderly. Nursing Researc; 1997.

26. Sánchez, Beatriz. Dimensión espiritual del cuidado de enfermería en situaciones de cronicidad y muerte, Bogotá, (en prensa); 2004.

27. Sulmasy DP.. Spiritual issues in the care of dying patients: "...it's okay between me and god". JAMA.; 2006.

28. Sulmasy DP.. The Rebirth of the Clinic. An Introduction to Spirituality in Health Care. Georgetown University Press, Washington DC.; 2006. 